\title{
New Delhi metallo- $\beta$-lactamase-1: detection and prevention
}

\author{
Timothy R. Walsh PhD
}

See related research article by Kus and colleagues on page 1257 and at www.cmaj.ca/lookup/doi/10.1503/cmaj.110477.

Competing interests:

Timothy Walsh has received grants from European

Commission and Wellcome

Trust, he has received payment for lectures from bioMérieux, and he has been reimbursed for travel expenses by Pfizer.

This article was solicited and has not been peer reviewed.

Correspondence to: Timothy R. Walsh,

t.r.walsh@uq.edu.au

CMAJ 2011. DOI:10.1503 /cmaj.111100

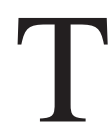
he advent of New Delhi metallo- $\beta$ lactamase-1 (NDM-1) as a new mechanism of microbial resistance went mostly unmarked, despite it being plasmidmediated, highly transferable and able to confer resistance to most antibiotics (with the exceptions of colistin and tigecycline). ${ }^{1,2}$ However, after the Indian subcontinent was linked to the spread of organisms producing NDM-1, and in response to the resulting reaction from India's Government, NDM-1 became an overnight global phenomenon. ${ }^{3,4}$ Thus far, most cases of infection or colonization with NDM-1producing bacteria have originated in southern Asia, most often in India. It is now clear that NDM-1 is firmly established in India, both in the hospitals and in the community. At the community level, the situation is exacerbated by the poor sanitary conditions with which many Indians must live on a daily basis. ${ }^{5}$

From a diagnostic and microbiologic perspective, NDM-1 is not easily identified in clinical bacteria. Some methods of detection lack the sensitivity necessary for the rapid and assured detection of NDM-1 that facilitates antibiotic stewardship and the introduction of measures to control the spread of infection. ${ }^{6}$

The story of NDM-1 is still in its infancy; however, it has already eclipsed the Klebsiella pneumoniae carbapenemases in terms of the number of countries in which it is found. In addition, because of the promiscuous nature of the plasmids containing the NDM-1 gene, the enzyme has been found in many more species than have the K. pneumoniae carbapenemases. Furthermore, our knowledge of NDM-1 is compromised by poor case studies; additional information, such as carriage and persistence, is required.

In their article for the CMAJ, Kus and colleagues ${ }^{7}$ present two important but contrasting case studies. The first patient, an 86-year-old man, had no contact with India or southern Asia. The Morganella morganii isolated from a culture of the patient's urine showed sensitivity to meropenem based on results from the Vitek II automated susceptibility testing system (bioMérieux, France), but carbapenemases were not detected by the Modified Hodge Test. However, despite these results, the organism was NDM-1 positive. These data throw into considerable doubt the validity of some of the methods heavily relied upon by diagnostic laboratories for the detection of antibiotic resistance. ${ }^{6}$

That the patient had no primary contact with southern Asia (indeed, he had not travelled outside of Canada in the previous 10 years) may be touted as evidence by the people who wish to propagate the myth that NDM-1 is everywhere. ${ }^{8,9}$ However, the link between southern Asia and most NDM-1-producing isolates is overwhelming, and the spread of such bacteria to the international community, such that direct links to southern Asia cannot be confirmed, is inevitable.

Another important feature of this particular case is that the isolate could be found in the patient's urine five months after the original diagnosis, an unusual show of persistence. Clearly, the isolate is not overtly pathogenic; however, it is able to persist in unusual environments, indicating a possible link between antibiotic resistance and persistence rather than pathogenicity. Unfortunately, samples of the patient's stool were not taken, which may have shown the presence of the M. morganii strain or other isolates that could have conferred the NDM-1 gene to the patient's natural flora.

In the second case, an NDM-1-producing isolate of Providencia rettgeri caused a urinary tract infection in a 71-year-old woman who had recently travelled to India. For this patient, the link to southern Asia is indisputable. The patient

All editorial matter in CMAJ represents the opinions of the authors and not necessarily those of the Canadian Medical Association. 
presented to hospital in Toronto, where samples of her urine were taken in which the NDM-1producing $P$. rettgeri was detected. Again, a follow-up culture of the patient's urine several months later showed that the organism was persisting, serving as another example of antibiotic resistance being linked to persistence rather than to pathogenicity. ${ }^{10}$ As in the previous case, because samples of this patient's stool were not taken, fecal carriage and in situ transfer of the NDM-1 gene could not be investigated.

Both patients were colonized by or infected with bacteria not usually associated with NDM1 , suggesting that the NDM-1 gene pool has now established itself in species other than Escherichia coli and K. pneumoniae. NDM-1producing bacteria typically show a high level of multidrug resistance or near pan-drug resistance. Although the organisms isolated from both of these patients were resistant to colistin, treatment with that antibiotic would not have been appropriate anyway. In fact, the only drug available to treat infections caused by NDM-1-producing organisms is tigecycline, which could not have been used because it is not indicated for the treatment of urinary tract infections.

Both of the patients described by Kus and colleagues ${ }^{7}$ were asymptomatic, so treatment of their infections was not necessary. However, if the patients had been symptomatic, their urinary tract infections would have been untreatable - Providencia and Morganella species are intrinsically resistant to colistin. Thankfully, neither of these organisms are prominent pathogens in the West, although we have very little data on their prevalences in southern Asia. A previous study by Walsh and colleagues ${ }^{5}$ suggests that the NDM-1 gene pool in southern Asia (i.e., carriage in normal flora) could be as high as $20 \%$. It is therefore possible that, owing to poor sanitation, NDM-1producing organisms could further spread throughout southern Asia, causing an increase in the prevalence of conditions such as cystitis. ${ }^{4.5}$

The examples reported by Kus and colleagues ${ }^{7}$ are but two of several cases of colonization or infection with NDM-1-producing organisms known to have occurred in Canada; for the foreseeable future, the Canadian medical community can brace itself for many more - whether they are linked to India or not. The spread of NDM-1 is not only due to its high rate of genetic transfer among unrelated bacterial species, but also to human factors such as travel, sanitation and food production and preparation. The rise of NDM-1 has caused us to rethink antibiotic resistance and the global spread of resistance mechanisms. As such, increased vigilance and appropriate surveillance are mandatory.

\section{References}

1. Deshpande P, Rodrigues C, Shetty A, et al. New Delhi Metallo$\beta$-lactamase (NDM-1) in Enterobacteriaceae: treatment options with carbapenems compromised. J Assoc Physicians India 2010; 58:147-9.

2. Yong D, Toleman MA, Giske CG, et al. Characterization of a new metallo- $\beta$-lactamase gene, bla(NDM-1), and a novel erythromycin esterase gene carried on a unique genetic structure in Klebsiella pneumoniae sequence type 14 from India. Antimicrob Agents Chemother 2009;53:5046-54.

3. Kumarasamy KK, Toleman MA, Walsh TR, et al. Emergence of a new antibiotic resistance mechanism in India, Pakistan, and the UK: a molecular, biological, and epidemiological study. Lancet Infect Dis 2010;10:597-602.

4. Walsh TR, Toleman MA. The new medical challenge: why NDM-1? Why Indian? Expert Rev Anti Infect Ther 2011;9:137-41.

5. Walsh TR, Weeks J, Livermore DM, et al. Dissemination of NDM-1 positive bacteria in the New Delhi environment and its implications for human health: an environmental point prevalence study. Lancet Infect Dis 2011;11:355-62.

6. Nordmann P, Poirel L, Carrer A, et al. How to detect NDM-1 producers. J Clin Microbiol 2011;49:718-21.

7. Kus JV, Tadros M, Simor A, et al. New Delhi metallo- $\beta$-lactamase-1: local acquisition in Ontario, Canada, and challenges in detection. CMAJ 2011;183:1257-61.

8. Bhaskar E. New Delhi metallo- $\beta$-lactamase 1. Lancet Infect Dis. 2010, 10:749; author reply 752-744.

9. Sirohi B. New Delhi metallo- $\beta$-lactamase 1. Lancet Infect Dis 2010;10:750, author reply 752-4

10. Poirel L, Hombrouck-Alet C, Freneaux C, et al. Global spread of New Delhi metallo- $\beta$-lactamase 1. Lancet Infect Dis 2010;10:832.

Affiliations: Timothy R. Walsh is with the Section of Medical Microbiology, School of Medicine, Cardiff University, Heath Park, Cardiff, Wales; and with the Clinical Centre for Research, University of Queensland, Herston, Queensland, Australia. 